\title{
Changes in Gene Expression and Neuroinflammation in the Hippocampus after Focal Brain Ischemia: Involvement in the Long-Term Cognitive and Mental Disorders
}

\author{
Galina T. Shishkina ${ }^{1, a *}$, Tatiana S. Kalinina ${ }^{1}$, Natalia V. Gulyaeva ${ }^{2}$, \\ Dmitry A. Lanshakov ${ }^{1}$, and Nikolay N. Dygalo ${ }^{1}$ \\ ${ }^{1}$ Institute of Cytology and Genetics, Siberian Branch of the Russian Academy of Sciences, 630090 Novosibirsk, Russia \\ ${ }^{2}$ Institute of Higher Nervous Activity and Neurophysiology, Russian Academy of Sciences, 117485 Moscow, Russia \\ ${ }^{a}$ e-mail: gtshi@bionet.nsc.ru
}

Received October 14, 2020

Revised December 23, 2020

Accepted January 9, 2021

\begin{abstract}
Ischemic brain injuries are accompanied by the long-term changes in gene expression in the hippocampus, the limbic system structure, involved in the regulation of key aspects of the higher nervous activity, such as cognitive functions and emotions. The altered expression of genes and proteins encoded by them may be related to the development of postischemic psycho-emotional and cognitive disturbances. Activation of neuroinflammation following stroke in the hippocampus has been suggested to play an essential role in induction of long-lasting consequences. Identification of changes in the gene expression patterns after ischemia and investigation of the dynamics of these changes in the hippocampus are the necessary first steps toward understanding molecular pathways responsible for the development of post-stroke cognitive impairments and mental pathologies.
\end{abstract}

DOI: $10.1134 / \mathrm{S} 0006297921060043$

Keywords: focal brain injuries, neuroinflammation, hippocampus, gene expression, cognitive impairment, depression

\section{INTRODUCTION}

Acute disruption of blood supply to the brain often impairs cognitive functions and triggers development of mental disorders, such as depression and anxiety [1-4]. These associated with stroke pathologies not only present problems in itself, but also negatively affect the poststroke rehabilitation, promote disability, and increase patient mortality [5,6]. Despite specificity of the pathways involved in the development of such pathologies in comparison with, for example, major depressive disorder [4], traditional antidepressants were found to be effective in extending survival of stroke patients for up to ten years on average [2]. Elucidation of mechanisms of strokeinduced pathologies might facilitate the search for the therapeutic targets in the development of new efficient strategies for the treatment of mental disorders.

Abbreviations: LPS, lipopolysaccharide; MCAO, middle cerebral artery occlusion.

* To whom correspondence should be addressed.
Hippocampus is considered to be the key brain structure involved in memory and learning. In particular, neurogenesis in the subgranular zone of the hippocampus is often associated with both cognitive and psycho-emotional functions; hence, secondary (delayed) post-stroke damage of this structure could be essential in the development of cognitive and mental disorders [7, 8]. The molecular mechanisms of such disorders developing after ischemic brain injury could involve long-term changes in the gene expression patterns in various brain structures, including limbic system (primarily, the hippocampus). Selective susceptibility of animal hippocampus to the development of neuroinflammation after exposure to various stress factors has been reported [9]. On the other hand, neuroimmune activation by administration of bacterial lipopolysaccharide (LPS) to animals causes longlasting impairment of cognitive functions [10] and changes in the gene expression patterns in the hippocampus [11], which suggests that neuroinflammation-related factors are the key mediators between ischemia and delayed alterations in the expression of genes essential for pathogenesis of post-stroke disturbances. The objective of 
this review was comparative analysis of available published data on the acute and delayed changes in the gene expression in the hippocampus after ischemic stroke and activation of neuroinflammation resulting in long-lasting cognitive and psycho-emotional impairments.

\section{MODELING OF ISCHEMIC DAMAGE AND NEUROINFLAMMATION IN RODENTS}

Cellular and molecular mechanisms of post-stroke psychiatric and cognitive disorders are commonly studied in the animal stroke models. For example, ischemic stroke can be induced by thromboembolic clots or administration of vasoconstrictive agents (e.g., endothelin 1) [12]. Almost $85 \%$ cases of stroke in humans are associated with ischemic damage [2] caused by the middle cerebral artery occlusion (MCAO). For this reason, transient $\mathrm{MCAO}$ is the most often procedure to induce stroke in rodents. In the rat MCAO model, focal ischemic damage of the neocortex initiated accumulation of corticosterone and interleukin (IL)-1 $\beta$ in the hippocampus within the first days after the stroke, the effect being most pronounced in the ventral region [13]. Comparison of these results with the changes in plasma corticosterone levels after MCAO indicates that one of the factors in the delayed damage of the hippocampus could be excessive concentration of circulating glucocorticoid hormone interacting with the hippocampal receptors. The impact of stress induced by deterioration of the physical state and forced social isolation after stroke has been suggested as an additional cause of delayed post-stroke psychological disorders, in particular, depression [2]. That is why in some cases, stress factors are introduced in addition to MCAO to model the respective pathologies in animals [14, 15].

Ischemic damage is accompanied by the activation of peripheral and central immune systems. However, activation of the peripheral immune system in the available ischemia models may not fully reflect the human inflammatory response following ischemic stroke. Thus, it was established that in humans, stroke altered expression of a number of genes, while some their mouse orthologs were close to random in matching changes in the expression of human genes, which, according to the authors, was due to the different leukocyte composition in the blood of humans and rodents [16]. In addition to the expansion of techniques for experimental induction of stroke, is necessary to use approaches for specific activation of proinflammatory processes in order to elucidate their role in the development of delayed post-stroke psychopathologies. The most common method is peripheral or central administration of bacterial lipopolysaccharide (LPS) [1719]. Despite the fact that the similarity between the molecular mechanisms of LPS-induced behavioral changes in animals and humans is currently poorly understood, LPS introduction is still considered as a useful technique for modeling neuroinflammation-induced changes in the central nervous system associated with the development of mental pathologies [19].

An active search for neurobiological correlates of psychopathological symptoms is currently underway in order to elucidate the mechanisms of development of post-stroke pathologies and to expand a repertoire of therapeutic interventions. Analysis of gene expression dynamics in the central nervous system after ischemic attack can facilitate identification of key components involved in this pathological process, as well as of new therapeutic targets. Gene expression in the hippocampus in the periods delayed from ischemia has been studied predominantly for separate genes. The use of genomewide transcriptome analysis of this brain structure is limited to the early stages after the experimental ischemic attack. Analysis of gene expression after exposure to LPS could be informative for elucidating the role of neuroinflammation in the delayed alterations in gene expression.

\section{RAPID RESPONSES OF THE HIPPOCAMPUS TO THE ISCHEMIC DAMAGE}

It is assumed that the processes occurring directly in the area of ischemic damage (increased production of reactive oxygen species and nitric oxide, glutamatergic excitotoxicity, neuroinflammation) exacerbate the damaging effect of stroke on the cells in the affected region and extend this effect to the structures that do not directly receive blood supply through the damaged artery. The emergence of activated microglial cells in the hippocampus, predominantly in the CA 3 region and dentate gyrus [20], indicates, according to the authors, an association of the activation of these cells with the damage of the entorhinal cortex followed by recruitment of the performant path fibers from the entorhinal cortex into the hippocampus, as well as hippocampal commissural fibers.

Mechanisms of ischemia damage in the hippocampal cells. An increased content of extracellular glutamate and resulting hyperactivation of $\mathrm{N}$-methyl-D-aspartate (NMDA) receptors followed by the massive calcium influx into the cells, are considered as the key factors in the damage of hippocampal neurons. As established using microdialysis, the extracellular level of glutamate in the rat hippocampus increased within the first minutes of ischemia [21]. The first evidences of the so-called delayed death of neurons in this structure were detected later than in the cortex and the striatum [22], as a rule, between $12 \mathrm{~h}$ and seven days after the ischemia, with the peak levels on day 4 [23]. Apparent signs of apoptosis induction were observed in the rat hippocampus one day after MCAO [24]. The death of hippocampal cells via apoptosis was confirmed by the decrease in the expression of anti-apoptotic protein Bcl-2, as well as by the upregulated expres- 
sion of the pro-apoptotic protein BAX [25] and the apoptosis-executing enzyme caspase- 3 in the hippocampus $[22,26]$. The increase in the number of apoptotic cells was accompanied with pronounced changes in the expression of mRNAs for the NMDA receptor subunits [27].

During the period of excitotoxic damage associated with stroke, the soma and dendrites of cells swell rapidly, which could result in the activation of volume-regulated anion channels (VRACs) helping restore cell volume. However, chronically swelling-activated VRACs are capable of direct glutamate release, aggravating neuronal excitotoxic death by further activation of NMDA receptors, as was discovered recently in the CA1 region of the mouse hippocampus after MCAO [28]. Because of the glutamatergic neurotransmission dysfunction, the response of astrocytes to ischemia becomes essential. These cells remove released glutamate from the extracellular space, thereby alleviating its excitotoxic effect. The activity of astrocytes increases after stroke; however, the consequences can be still negative due to a significant increase in the synthesis of the calcium-binding protein $\mathrm{S} 100 \mathrm{~B}$, which is predominately expressed in the astrocytes. In low concentrations, SB100 positively affects brain cells, but in high concentrations, it suppresses the synthesis of the glutamate transporter and the antioxidant glutathione. As a result, reverse glutamate uptake becomes insufficient, and glutamate is accumulated in the extracellular space, which, in turn, promotes neuronal death. Suppression of the S100B synthesis facilitates the antioxidant defense and improves neuron survival after stroke [29]. Transcriptome analysis conducted one day after MCAO revealed elevated expression of the gene coding for $\mathrm{S} 100 \mathrm{a} 9$ (calgranulin $\mathrm{B}$ ), a member of the calcium-binding proteins (S100) group, but the significance of this phenomenon is still to be understood [26].

MCAO-induced increase in the content of vacuolar protein sorting 4 homologue B (VPS4B) should also be mentioned among the mechanism of ischemia-related neuronal death in the hippocampus. This protein is predominately located in the neuron cytoplasm. It belongs to the group of transport ATPases associated with secretion and internalization of exosomes, intracellular protein transport, and cell proliferation. The level of VPS4B protein in the CA1 region of the hippocampus reached its peak three days after MCAO. This increase was accompanied by a reliable activation of the expression of caspase3, which co-localizes with VPS4B in the cells [30]. Suppression of VPS4B expression with a small interfering RNA (siRNA) in the culture of PC12 cells caused a decrease in the level of active caspase- 3 and reduction of cell death induced by oxygen and glucose deficit.

Activation of neuroinflammation. The products of cell death caused by brain ischemia, such as hyaluronic acid, high-mobility group box 1 protein, mRNA, heat shock proteins, etc., activate Toll-like receptors (TLRs), result- ing in the stimulation of secretion of proinflammatory cytokines including TNF- $\alpha$ (tumor necrosis factor alpha), IL-1 $\alpha$, IL-1 $\beta$, IL-6, and interferon (IFN) [31]. Induced neuroinflammation is manifested by the amoeboid-like morphological transformation of microglia, the resident brain immune cells, as well as by secondary increase in the levels of proinflammatory cytokines produced by the activated microglia and astrocytes in the CNS [32].

Activated microglial cells were found in the rat hippocampus two days after MCAO [20]. It must be mentioned that microglia plays a dual role in the CNS [33]. One of its important functions is neuroprotection via secretion of anti-inflammatory cytokines and neurotrophic factors, primarily, brain-derived neurotrophic factor (BDNF), thus facilitating neuron survival in the penumbra [34]. Expression of the $\alpha 7$ subunit-containing nicotine acetylcholine receptor, crucial for the regulation of the neuroprotective function of microglia, decreased in response to neuroinflammation [35]. Protective phagocytic capacity of the microglia is normally low, but it increases under inflammatory conditions. Furthermore, it was suggested that hyperactivation or chronic activation of the microglia results in the loss of its classic neuroprotective activity, which is manifested by its decreased involvement in the formation and remodeling of synapses and leads to the development of neurodegenerative diseases, including those associated with impaired mental and cognitive functions [32]. One of the key transcription factors initiating the proinflammatory response to ischemia and LPS in the microglia is nuclear factor kappa $\mathrm{B}(\mathrm{NF}-\kappa \mathrm{B})$ [36]. NF- $\kappa \mathrm{B}$ and cAMP response elementbinding protein (CREB) are responsible for switching the phenotypes of microglial cells associated with secretion of proinflammatory cytokines (M1) and neurotrophic factors (M2) [37].

Changes in gene expression. Transcriptome analysis of the hippocampus carried out with DNA microchips or by RNA sequencing within the first 4.5-6 $\mathrm{h}$ and one day after MCAO revealed association of gene expression with various cellular and neurobiological events. The methodological differences in the published studies include different duration of occlusion $(90,120$, or $180 \mathrm{~min})$ and sample size (hippocampus only [25] or in complex with other subcortical structures [26]). Nevertheless, the data presented in these studies demonstrate common trends in the expression of genes associated primarily with the cell death and activation of neuroinflammation [2426, 38].

The number of hippocampal genes with altered expression significantly increased from the first hours until $24 \mathrm{~h}$ after ischemia-reperfusion (from 496 genes at $4.5 \mathrm{~h}$, to 1939 genes at $24 \mathrm{~h}$ ) [26]. Different sets of genes were suppressed/activated at different time points, presumably with different physiological consequences. In particular, genes coding for proteins of the hypoxia- 
inducible factor (HIF) signaling cascade (e.g., HIF-1) were upregulated within first hours after ischemia-reperfusion. Taking into account the well-known fact that HIFs facilitate expression of the vascular endothelial growth factor (VEGF) and glycolytic enzymes, upregulated expression of these proteins can be essential for metabolism maintenance under conditions of deficit of resources and reflects fast activation of adaptive response to the damage. At the same time, expression of genes associated with protein phosphorylation was noticeably decreased [38]. The genes with the most significant changes in expression $24 \mathrm{~h}$ after ischemia-reperfusion participate in inflammation, oxidative phosphorylation, endoplasmic reticulum stress, apoptosis, autophagy, and other metabolic processes.

It was also found that the transcriptional profiles of the CA1 and CA3 regions in the hippocampus of intact mice were different [39]. One day after ischemia/hypoxia, these regional differences were much less pronounced for the majority of genes. According to the authors, this was associated with the general activation of transcription. The list of genes with altered expression in both regions included those coding for proinflammatory factors and genes involved in stress response. Cell death and activation of neuroinflammation are among the most important events at the early stages after ischemia.

\section{ROLE OF NEUROINFLAMMATION IN POST-STROKE DISORDERS}

Post-stroke mental pathologies are often associated with cognitive impairments and mood disorders [1-4]. Considering that hippocampus plays an important role in the processing of spatial information and memory, as well as in the control of psycho-emotional state, the role of hippocampal changes, including neuroinflammation in the development of these consequences of stroke attracts a lot of attention of researchers.

Cognitive disturbances following ischemic stroke are manifested by the loss of memory and concentration, as well as speech impairments. Studies in rodents have shown that attenuation of neuroinflammation in the hippocampus after MCAO, for example, by exposure of animals to an enriched environment [40] or administration of insulin-like growth factor 1 [41] improves ischemiainduced cognitive deficits. On the contrary, activation of neuroinflammation by LPS resulted in memory loss, which was observed seven days [42] and even ten months [43] after a single administration of endotoxin, and reliably correlated with the increased levels of the proinflammatory cytokines TNF- $\alpha$ and IL- $1 \beta$ in the hippocampus.

Association of pro-inflammatory factors with the development of depression and anxiety has been confirmed by multiple clinical observations, such as psy- chopathology development in mentally healthy volunteers after stimulation of pro-inflammatory response with LPS [44], as well as during therapy with pro-inflammatory cytokines [45] or in patients with chronic inflammatory diseases [46]. Either single or chronic peripheral administration of LPS to animals in the experimental trials resulted in the emergence of symptoms of depressionlike behavior [47] and anxiety $24 \mathrm{~h}$ after the exposure $[48,49]$. Repeated intracerebroventricular infusion of LPS within five days (days 1, 3, 5) also induced depression-like behaviors in rats tested one day after the last infusion. The mRNA expression of TNF- $\alpha$ and IL-1 $\beta$ was increased after LPS, particularly in the hippocampus, which may have contributed to the behavioral alterations, as suggested by the authors [50]. The knockdown of IL$1 \beta$ expression in the hippocampus with a virus-encoded shRNA reduced the signs of the depression-like behavior and anxiety and alleviated memory loss induced by LPS [51]. Administration of tetracycline antibiotic minocycline to mice with ischemia interfered with neuroinflammation and alleviated depression-like behavior and anxiety in the animals [52].

Despite the fact that the published data indicate an important role of neuroinflammation in the pathogenesis of post-stroke disorders, molecular mechanisms by which pro-inflammatory factors affect cognitive and psychoemotional function are still poorly understood. The study on the gene expression in the hippocampus and the synthesis of the corresponding protein products for an extended period of time following ischemic attack revealed multiple changes involving immune and neurotransmitter systems, inflammation, neurodegeneration, neuroprotection, lipid metabolism, intra- and intercellular signaling, and a number of other cellular functions [53]. The processes that play an important role in the development of post-stroke pathologies associated with neuroinflammatory response include accumulation of $\beta$ amyloid and increased phosphorylation of tau protein, reduction in neuroplasticity, neurodegeneration, and changes in the activity of the serotoninergic system.

Beta-amyloid and tau protein. Post-stroke accumulation of $\beta$-amyloid and tau protein in the hippocampal cells induced by the initial activation of neuroinflammatory processes is one of the signs of long-term neuroinflammation developing after ischemic attack [54]. Studies in the animals conducted within 2 to 30 days after ischemia/reperfusion demonstrated dysregulation of expression of genes coding for the amyloid precursor protein, $\alpha-, \beta-$, and $\gamma$-secretases, and tau protein [55]. Some researchers relate $\beta$-amyloid accumulation and tau protein hyperphosphorylation in the brain [56], which are typical symptoms of Alzheimer's disease, to the progressive decrease in the cognitive functions after the stroke $[54,57]$. According to the prospective study [1], 17-92\% stroke patients were diagnosed with mild cognitive impairments 3 months after the ischemic attack, and the 
percentage of patients with dementia increased to $48 \% 25$ years after the stroke [1]. In animal experiments, cognitive function impairments evaluated 14 days after MCAO were accompanied by the increased phosphorylation of tau protein (at T205 and S396) in the hippocampus [41].

Inflammation, neurodegenerative changes, accumulation of $\beta$-amyloid, and phosphorylation of tau protein are associated with the cognitive impairments and dementia [54]. Neuroinflammation induces amyloid accumulation by promoting its synthesis from the precursor protein by secretases and/or via decreased secretion through various systems, including glymphatic system [57]. In addition to neuroinflammation, accumulation of tau protein after ischemic damage could be related to other mechanisms, such as activation of glutamatergic system. Thus, no increase in the amount of the hyperphosphorylated tau protein in the hippocampus was observed on day 10 after MCAO when the glutamatergic pathway from the entorhinal cortex to the hippocampus was disrupted [56].

Upregulated expression of the $\beta$-amyloid and tau protein genes is associated with the induction of neurodegeneration, which is assumed to be responsible for the cognitive impairments. Analysis of the hippocampus in patients with the post-ischemic dementia revealed a significant decrease in the neuron volume and density in the CA1 field [58]. At the same time, the data on the impact of amyloid pathology on the post-stroke neurodegeneration and cognitive impairments are scarce. While association between the post-stroke neuroinflammation and amyloid accumulation has been demonstrated in animal models, it has not been studied in humans, in particular, in the human hippocampus. Recently, an attempt has been made to investigate stroke patients with impaired cognitive functions in order to establish an association between the post-stroke neuroinflammation, $\beta$-amyloid deposition, and neurodegeneration in the cortex. However, no association between these parameters was observed in the patients seven years after the stroke [59], which indicated that further studies are required, including those at the molecular and genetic levels.

Neurogenesis and regulators of neuroplasticity. Neurodegeneration associated with the post-stroke cognitive and psycho-emotional disorders could be caused by impairments in neuroplasticity, which is modulated in large part by neurotrophic factors (e.g., BDNF). Reduction of learning ability and memory loss in adult rats four weeks after MCAO were accompanied by the disruption of the synaptic structure and decrease in the number of synaptic vesicles, as well as by the increased BDNF levels in the hippocampus. The expression of NMDAR1, on the other hand, was significantly lower than in the control [60]. Changes in the BDNF expression could depend on the time after the stroke, as well as on the effects of other stress factors. For example, augmentation of depression-like behavior in adult rats after
MCAO combined with isolation and chronic unpredictable mild stress during six weeks was accompanied by the downregulation of BDNF expression in the CA1 region of the hippocampus [61].

The intensity of neurogenesis, which takes place in the subgranular zone of the hippocampus for the entire lifetime, is important for the memory formation. It was shown that the age-related memory loss is quantitatively associated with a decrease in the rate of neurogenesis [62]. Surprisingly, post-stroke mice demonstrated activation of hippocampal neurogenesis that was maintained for one month after the stroke and correlated with the disruption of contextual and spatial memory in the animals [63]. Activation of neurogenesis depended on the duration of the ischemic episode; for example, it was more significant 28 days after 2-h ischemic attack in comparison with the 30-min MCAO, but did not depend on the extent of cell death in the hippocampus or on the extent of cortex damage [64]. Many researchers believe that despite its activation, post-stroke neurogenesis in the hippocampus could be an aberrant process, both functionally and structurally. For example, one of the causes of memory loss could be an altered morphology of the newborn granular cells [65]. Moreover, stroke can shift neurogenesis towards astrogliogenesis [66], which theoretically increases the number of astrocytes and the content of pro-inflammatory cytokines. Neurogenesis is controlled by numerous regulators, including glucocorticoids, neurotrophins, neurotransmitters, epigenetic factors. Changes in their activity could underlie the aberrant post-stroke neurogenesis and associated memory loss. It was suggested that activation of neurogenesis in the dentate gyrus of rats 28 days after 2-h MCAO was associated with the glutamatergic mechanisms, which was confirmed by complete inhibition of this activation by the NMDA receptor antagonists [64].

Serotoninergic system. The shift of tryptophan metabolism from the serotoninergic to the kynurenic pathway as a result of indoleamine 2,3-dioxygenase (IDO) activation [47] plays an important role in the prodepression effects of pro-inflammatory cytokines [47]. Peripheral administration of LPS activates IDO and induces depression-like behavior [67]. Such metabolic shift causes a deficit of tryptophan required for serotonin synthesis, resulting in the decreased content of this neurotransmitter in the brain and suppression of serotoninergic signaling, which is a risk factor for depressive disorders $[68,69]$. This reorganization also causes accumulation of kynurenine metabolites, mostly, quinolinic acid, that play their own role in the pathogenesis of depression [70]. Quinolinic acid is an agonist of NMDA receptors and, hence, facilitates excitotoxicity via increase in the extracellular glutamate concentration either by stimulating its release or by blocking its reverse uptake by astrocytes [71]. Mice lacking NMDA receptor GluN2A subunit in the prefrontal cortex and hippocampus did not develop 
depressive-like behavior in response to LPS administration [72]. The impact of the tryptophan metabolism shift from the serotoninergic to the kynurenic pathway on the emergence of post-stroke disorders has been mentioned in several studies. For example, development of depression-like state and memory loss 5-10 weeks after MCAO along with spatial restraint stress occurred on the background of increased IDO1 expression in the activated microglial cells in different brain regions including hippocampus [73]. Hence, elevated production of proinflammatory cytokines after ischemia could initiate and promote the pathological cascade of IDO activation, especially in the limbic and paralimbic areas, as well as development of post-stroke depression [73].

\section{ACTIVATION OF NEUROINFLAMMATION AS A POTENTIAL FACTOR IN THE LONG-TERM GENES INDUCTION IN THE HIPPOCAMPUS}

Neuroinflammation in the hippocampus could be maintained for a long time after ischemia. The number of microglial cells in the rat hippocampus was also increased on day seven after MCAO [20]; the levels of IL-1 $\beta$ and the number of IL-1 $\beta$-positive cells were significantly higher in these animals 28 days after the procedure [40]. Increased numbers of activated astrocytes and microglial cells in the rat hippocampus were revealed even two years after a 10-min global ischemia [54]. Cytosolic nucleotide-binding oligomerization domain (NOD)-like receptors (NLRs) could play a role in the long-term activation of neuroinflammation. It was recently shown [74] that MCAO caused a significant increase in the levels of the Nlrp10 mRNA and protein in the penumbra 7, 14, and 28 days after the procedure. Association of the increased Nlrp10 level with the neuroinflammation development in the hippocampus was confirmed by the weakening of the glial cell activation during ischemia in the Nlrp10 knockout mice. Compared with the wild-type animals, the number of GFAP- and Iba1-positive cells (astrocytes and microglia, respectively), as well as expression of proinflammatory cytokines in hippocampus of knockout mice were markedly decreased after MCAO. Astrocytes from Nlrp10 knockout mice demonstrated reduced response of the TLR-4/NF- $\kappa \mathrm{B}$ and NLRP12/ASC/caspase-1 signaling pathways to incubation with LPS.

The dynamics of changes in the parameters of neuroinflammation after LPS administration is similar to that observed after MCAO. For example, significant activation of microglia in the hippocampus was found 1-2 days after peripheral administration of a single dose of LPS $(1-2 \mathrm{mg} / \mathrm{kg})[32,75]$. The signs of neuroinflammation following LPS administration were also maintained for a long time. Analysis of the microglia response to the intra-hippocampal infusion of LPS $(10 \mu \mathrm{g})$ showed an increased number of activated microglial cells at all times
(6 and $24 \mathrm{~h}, 1$ and 4 weeks) after endotoxin administration [76].

Even short-term induction of inflammation can result in the long-lasting dysregulation of gene expression in the brain. Changes in the expression of 230 genes (183 - upregulated and 47 - downregulated) were observed in the hippocampus of adult male rats three months after the last of five LPS injections $(250 \mu \mathrm{g} / \mathrm{kg}$, administered within two weeks) [10]. The genes of the early response proteins and transcription factors (Egr2, Fosb, Fos, Npas4, Egr4, Junb), immune signaling (Ccl3), and transcription (Btg2) were among the genes with the most downregulated expression. The gene for transthyretin (Ttr), a protein that presumably participates in the development of amyloidosis [77] and oxidative stress [78], was among the genes with the most upregulated expression. In general, the data of the transcriptome analysis indicate that both development and maintenance of the post-stroke disorders could be mediated by the induction of long-lasting changes in the expression of genes involved in the control of cognitive and psychoemotional functions, as well as regulators of their transcription. Memory loss observed in rodents three months after introduction of a moderate dose of LPS $(250 \mu \mathrm{g} / \mathrm{kg}$; five injections, one every three days) [11] or five months after administration of a single high dose of this endotoxin $(1 \mathrm{mg} / \mathrm{kg})$ [79] imply such possibility. However, this suggestion has to be verified in experimental studies.

\section{CONCLUSIONS}

The data accumulated so far indicate an important role of neuroinflammation in the stroke pathogenesis and its possible involvement in provoking and maintaining the post-stroke cognitive impairments, as well as in the development of mental disorders through changes in the expression of regulatory genes in the hippocampus. This makes neuroinflammation a potential target for the therapeutic intervention. At the same time, some researchers [33] believe that the role of inflammation at different post-ischemia stages must be clarified, because brain resident immune cells can have both negative and positive effects on the neuronal survival. In particular, interventions aiming to suppress microglial cells at the wrong time could worsen the course of the disease. In this regard, identification of changes in the gene expression patterns after ischemia and investigation of dynamics of these changes in the hippocampus are the necessary first steps to understand molecular pathways responsible for the development of post-stroke cognitive and mental pathologies.

Funding. This work was supported by the Russian Science Foundation (project no. 20-64-47013) and by the State Budget Project 0259-2021-0015. 
Ethics declarations. The authors declare no conflict of interest in financial or any other sphere. This article does not contain any studies with human participants or animals performed by any of the authors.

Open access. This article is licensed under a Creative Commons Attribution 4.0 International License, which permits use, sharing, adaptation, distribution and reproduction in any medium or format, as long as you give appropriate credit to the original author(s) and the source, provide a link to the Creative Commons license, and indicate if changes were made. The images or other third party material in this article are included in the article's Creative Commons license, unless indicated otherwise in a credit line to the material. If material is not included in the article's Creative Commons license and your intended use is not permitted by statutory regulation or exceeds the permitted use, you will need to obtain permission directly from the copyright holder. To view a copy of this license, visit http://creativecommons.org/licenses/ by $/ 4.0 /$.

\section{REFERENCES}

1. Brainin, M., Tuomilehto, J., Heiss, W. D., Bornstein, N. M., Bath, P. M., et al. (2015) Post-stroke cognitive decline: an update and perspectives for clinical research, Eur. J. Neurol., 22, 229-238, doi: 10.1111/ene.12626.

2. Robinson, R. G., and Jorge, R. E. (2016) Post-stroke depression: a review, Am. J. Psychiatry, 173, 221-231, doi: 10.1176/appi.ajp.2015.15030363.

3. Pedroso, V. S. P., Brunoni, A. R., Vieira, É. L. M., Jorge, R. E., Lauterbach, E. C., and Teixeira, A. L. (2018) Early psychiatric morbidity in a Brazilian sample of acute ischemic stroke patients, Clinics (Sao Paulo), 73, e55, doi: 10.6061/clinics/2018/e055.

4. Baccaro, A., Wang, Y. P., Candido, M., Conforto, A. B., Brunoni, A. R., et al. (2019) Post-stroke depression and cognitive impairment: study design and preliminary findings in a Brazilian prospective stroke cohort (EMMA study), J. Affect. Disord., 245, 72-81, doi: 10.1016/j.jad. 2018.10.003.

5. De Mello, R. F., Santos, I. S., Alencar, A. P., Bensenor, I. M., Lotufo, P. A., and Goulart, A. C. (2016) Major depression as a predictor of poor long-term survival in a Brazilian stroke cohort (study of stroke mortality and morbidity in adults) EMMA study, J. Stroke Cerebrovasc. Dis., 25, 618-625, doi: 10.1016/j.jstrokecerebrovasdis.2015.11.021.

6. Paolucci, S., Iosa, M., Coiro, P., Venturiero, V., Savo, A., et al. (2019) Post-stroke depression increases disability more than $15 \%$ in ischemic stroke survivors: a case-control study, Front. Neurol., 10, 926, doi: 10.3389/fneur.2019. 00926.

7. Gulyaeva, N. V. (2019) Biochemical mechanisms and translational relevance of hippocampal vulnerability to distant focal brain injury: the price of stress response, Biochemistry (Moscow), 84, 1306-1328, doi: 10.1134/ S0006297919110087.

8. Gulyaeva, N. V. (2019) Functional neurochemistry of the ventral and dorsal hippocampus: stress, depression, demen- tia and remote hippocampal damage, Neurochem. Res., 44, 1306-1322, doi: 10.1007/s11064-018-2662-0.

9. Piskunov, A., Stepanichev, M., Tishkina, A., Novikova, M., Levshina, I., and Gulyaeva, N. (2016) Chronic combined stress induces selective and long-lasting inflammatory response evoked by changes in corticosterone accumulation and signaling in rat hippocampus, Metab. Br. Dis., 31, 445-454, doi: 10.1007/s11011-015-9785-7.

10. Tchessalova, D., and Tronson, N. C. (2020) Enduring and sex-specific changes in hippocampal gene expression after a subchronic immune challenge, Neuroscience, 428, 76-89, doi: 10.1016/j.neuroscience.2019.12.019.

11. Tchessalova, D., and Tronson, N. C. (2019) Memory deficits in males and females long after subchronic immune challenge, Neurobiol. Learn. Mem., 158, 60-72, doi: 10.1016/j.nlm.2019.01.003.

12. Fluri, F., Schuhmann, M. K., and Kleinschnitz, C. (2015) Animal models of ischemic stroke and their application in clinical research, Drug Des. Dev. Ther., 9, 3445-3454, doi: 10.2147/DDDT.S56071.

13. Onufriev, M. V., Freiman, S. V., Moiseeva, Yu. V., Stepanichev, M. Yu., Lazareva, N. A., and Gulyaeva, N. V. (2017) Accumulation of corticosterone and interleukin-1 $\beta$ in the hippocampus after focal ischemic damage of the neocortex: selective vulnerability of the ventral hippocampus, Neurochem. J., 11, 236-241, doi: 10.1134/S1819712417030084.

14. Kronenberg, G., Gertz, K., Heinz, A., and Endres, M. (2014) Of mice and men: modelling post-stroke depression experimentally, Br. J. Pharmacol., 171, 4673-4689, doi: 10.1111/bph.12775.

15. Xu, Y., and Liang, L. (2020) Vitamin D3/vitamin D receptor signaling mitigates symptoms of post-stroke depression in mice by upregulating hippocampal BDNF expression, Neurosci. Res., doi: 10.1016/j.neures.2020.08.002.

16. Sharp, F. R., and Jickling, G. C. (2014) Modeling immunity and inflammation in stroke: differences between rodents and humans? Stroke, 45, e179-180, doi: 10.1161/ STROKEAHA.114.005639.

17. Stepanichev, M., Dygalo, N. N., Grigoryan, G., Shishkina, G. T., and Gulyaeva, N. (2014) Rodent models of depression: neurotrophic and neuroinflammatory biomarkers, Biomed. Res. Int., 2014, 932757, doi: 10.1155/2014/ 932757.

18. Zhan, X., Cox, C., Ander, B. P., Liu, D., Stamova, B., et al. (2015) Inflammation combined with ischemia produces myelin injury and plaque-like aggregates of myelin, amyloid- $\beta$ and A $\beta$ PP in adult rat brain, J. Alzheimer's Dis., 46, 507-523, doi: 10.3233/JAD-143072.

19. Lasselin, J., Schedlowski, M., Karshikoff, B., Engler, H., Lekander, M., and Konsman, J. P. (2020) Comparison of bacterial lipopolysaccharide-induced sickness behavior in rodents and humans: relevance for symptoms of anxiety and depression, Neurosci. Biobehav. Rev., 115, 15-24, doi: 10.1016/j.neubiorev.2020.05.001.

20. Morioka, T., Kalehua, A. N., and Streit, W. J. (1993) Characterization of microglial reaction after middle cerebral artery occlusion in rat brain, J. Comp. Neurol., 327, 123-132, doi: 10.1002/cne.903270110.

21. Yang, Y., Li, Q., Miyashita, H., Yang, T., and Shuaib, A. (2001) Different dynamic patterns of extracellular glutamate release in rat hippocampus after permanent or 30-min transient cerebral ischemia and histological correlation, 
Neuropathology, 21, 181-187, doi: 10.1046/j.14401789.2001.00397.x.

22. Mrsis-Pelcic, J., Pilipovic, K., Pelcic, G., Vitezic, D., and Zupan, G. (2017) Decrease in oxidative stress parameters after post-ischaemic recombinant human erythropoietin administration in the hippocampus of rats exposed to focal cerebral ischaemia, Basic Clin. Pharmacol. Toxicol., 121, 453-464, doi: 10.1111/bcpt.12833.

23. Butler, T. L., Kassed, C. A., Sanberg, P. R., Willing, A. E., and Pennypacker, K. R. (2002) Neurodegeneration in the rat hippocampus and striatum after middle cerebral artery occlusion, Brain Res., 929, 252-260, doi: 10.1016/s00068993(01)03371-6.

24. Chung, J. Y., Yi, J. W., Kim, S. M., Lim, Y. J., Chung, J. H., and Jo, D. J. (2011) Changes in gene expression in the rat hippocampus after focal cerebral ischemia, $J$. Korean Neurosurg. Soc., 50, 173-178, doi: 10.3340/jkns. 2011.50.3.173.

25. Wang, C., Liu, M., Pan, Y., Bai, B., and Chen, J. (2017) Global gene expression profile of cerebral ischemia-reperfusion injury in rat MCAO model, Oncotarget, 8, 7460774622, doi: 10.18632/oncotarget. 20253.

26. Dergunova, L. V., Filippenkov, I. B., Stavchansky, V. V., Denisova, A. E., Yuzhakov, V. V., et al. (2018) Genomewide transcriptome analysis using RNA-Seq reveals a large number of differentially expressed genes in a transient MCAO rat model, BMC Genomics, 19, 655, doi: 10.1186/ s12864-018-5039-5.

27. Liu, Z., Zhao, W., Xu, T., Pei, D., and Peng, Y. (2010) Alterations of NMDA receptor subunits NR1, NR2A and NR2B mRNA expression and their relationship to apoptosis following transient forebrain ischemia, Brain Res., 1361, 133-139, doi: 10.1016/j.brainres.2010.09.035.

28. Zhou, J. J., Luo, Y., Chen, S. R., Shao, J. Y., Sah, R., and Pan, H. L. (2020) LRRC8A-dependent volume-regulated anion channels contribute to ischemia-induced brain injury and glutamatergic input to hippocampal neurons, Exp. Neurol., 332, 113391, doi: 10.1016/j.expneurol.2020.113391.

29. Cordeiro, J. L., Neves, J. D., Vizuete, A. F., Aristimunha, D., Pedroso, T. A., et al. (2020) Arundic Acid (ONO-2506), an inhibitor of S100B protein synthesis, prevents neurological deficits and brain tissue damage following intracerebral hemorrhage in male Wistar rats, Neuroscience, 440, 97-112, doi: 10.1016/j.neuroscience. 2020.05.030.

30. Cui, G., Wang, Y., Yu, S., Yang, L., Li, B., et al. (2014) The expression changes of vacuolar protein sorting 4B (VPS4B) following middle cerebral artery occlusion (MCAO) in adult rats brain hippocampus, Cell. Mol. Neurobiol., 34, 8394, doi: 10.1007/s10571-013-9989-5.

31. Kumar, V. (2019) Toll-like receptors in the pathogenesis of neuroinflammation, $J$. Neuroimmunol., 332, 16-30, doi: 10.1016/j.jneuroim.2019.03.012.

32. Bowyer, J. F., Sarkar, S., Burks, S. M., Hess, J. N., Tolani, S., et al. (2020) Microglial activation and responses to vasculature that result from an acute LPS exposure, Neurotoxicology, 77, 181-192, doi: 10.1016/j.neuro.2020. 01.014 .

33. Jayaraj, R. L., Azimullah, S., Beiram, R., Jalal, F. Y., and Rosenberg, G. A. (2019) Neuroinflammation: friend and foe for ischemic stroke, J. Neuroinflammation, 16, 142, doi: 10.1186/s12974-019-1516-2.
34. Jiang, M. Q., Zhao, Y. Y., Cao, W., Wei, Z. Z., Gu, X., et al. (2017) Long-term survival and regeneration of neuronal and vasculature cells inside the core region after ischemic stroke in adult mice, Brain Pathol., 27, 480-498, doi: 10.1111/bpa.12425.

35. Nakamura, Y., Kimura, S., Takada, N., Takemura, M., Iwamoto, M., et al. (2020) Stimulation of toll-like receptor 4 downregulates the expression of $\alpha 7$ nicotinic acetylcholine receptors via histone deacetylase in rodent microglia, Neurochem. Int., 138, 104751, doi: 10.1016/ j.neuint.2020.104751.

36. Xu, H., Qin, W., Hu, X., Mu, S., Zhu, J., et al. (2018) Lentivirus-mediated overexpression of OTULIN ameliorates microglia activation and neuroinflammation by depressing the activation of the NF- $\mathrm{BB}$ signaling pathway in cerebral ischemia/reperfusion rats, J. Neuroinflammation, 15, 83, doi: 10.1186/s12974-018-1117-5.

37. Xia, C. Y., Zhang, S., Gao, Y., Wang, Z. Z., and Chen, N. H. (2015) Selective modulation of microglia polarization to M2 phenotype for stroke treatment, Int. Immunopharmacol., 25, 377-382, doi: 10.1016/j.intimp. 2015.02.019.

38. Liu, X., Wang, Q., Cui, Y., Li, X., and Yang, H. (2020) Indepth transcriptomic and proteomic analyses of the hippocampus and cortex in a rat model after cerebral ischemic injury and repair by Shuxuetong (SXT) injection, J. Ethnopharmacol., 249, 112362, doi: 10.1016/j.jep.2019. 112362.

39. Newrzella, D., Pahlavan, P. S., Krüger, C., Boehm, C., Sorgenfrei, O., et al. (2007) The functional genome of CA1 and CA3 neurons under native conditions and in response to ischemia, BMC Genomics, 8, 370, doi: 10.1186/14712164-8-370.

40. Zhang, X., Yuan, M., Yang, S., Chen, X., Wu, J., et al. (2020) Enriched environment improves post-stroke cognitive impairment and inhibits neuroinflammation and oxidative stress by activating Nrf2-ARE pathway, Int. J. Neurosci., doi: 10.1080/00207454.2020.1797722.

41. Yang, W., Li, G., Cao, K., Ma, P., Guo, Y., et al. (2020) Exogenous insulin-like growth factor 1 attenuates acute ischemic stroke-induced spatial memory impairment via modulating inflammatory response and tau phosphorylation, Neuropeptides, 83, 102082, doi: 10.1016/j.npep.2020. 102082.

42. Kawano, T., Morikawa, A., Imori, S., Waki, S., Tamura, T., et al. (2014) Preventive effects of multisensory rehabilitation on development of cognitive dysfunction following systemic inflammation in aged rats, J. Anesth., 28, 780-784, doi: 10.1007/s00540-013-1786-2.

43. Bossu, P., Cutuli, D., Palladino, I., Caporali, P., Angelucci, F., et al. (2012) A single intraperitoneal injection of endotoxin in rats induces long-lasting modifications in behavior and brain protein levels of TNF- $\alpha$ and IL-18, J. Neuroinflamm., 9, 101, doi: 10.1186/1742-2094-9-101.

44. Reichenberg, A., Yirmiya, R., Schuld, A., Kraus, T., Haack, M., et al. (2001) Cytokine-associated emotional and cognitive disturbances in humans, Arch. Gen. Psychiatry, 58, 445-452, doi: 10.1001/archpsyc.58.5.445.

45. Schiepers, O. J., Wichers, M. C., and Maes, M. (2005) Cytokines and major depression, Prog. Neuropsychopharmacol. Biol. Psychiatry, 29, 201-217, doi: 10.1016/j.pnpbp.2004. 11.003 . 
46. Rukavishnikov, G. V., Kibitov, A. O., Mazo, G. E., and Neznanov, N. G. (2019) Genetic determinism of comorbidity of depression and somatic diseases, Neurosci. Behav. Physiol., 119, 89-96, doi: 10.17116/jnevro201911901189.

47. Dantzer, R., O’Connor, J. C., Freund, G. G., Johnson, R. W., and Kelley, K. W. (2008) From inflammation to sickness and depression: when the immune system subjugates the brain, Nat. Rev. Neurosci., 9, 46-56, doi: 10.1038/ nrn2297.

48. Shishkina, G. T., Bannova, A. V., Komysheva, N. P., and Dygalo, N. N. (2019) Doxycycline attenuates anxiety and microglia activation induced by repeated lipopolysaccharide, Eur. Neuropsychopharmacol., 29, S179-S180, doi: 10.1016/j.euroneuro.2019.09.276.

49. Shishkina, G. T., Bannova, A. V., Komysheva, N. P., and Dygalo, N. N. (2020) Anxiogenic-like effect of chronic lipopolysaccharide is associated with increased expression of matrix metalloproteinase 9 in the rat amygdala, Stress, 23, 708-714, doi: 10.1080/10253890.2020.1793943.

50. Guan, X. T., Lin, W. J., and Tang, M. M. (2015) Comparison of stress-induced and LPS-induced depressive-like behaviors and the alterations of central proinflammatory cytokines mRNA in rats, Psych. J., 4, 113-122, doi: $10.1002 /$ pchj.87.

51. Li, M., Li, C., Yu, H., Cai, X., Shen, X., et al. (2017) Lentivirus-mediated interleukin-1 $\beta$ (IL-1 $\beta$ ) knock-down in the hippocampus alleviates lipopolysaccharide (LPS)induced memory deficits and anxiety- and depression-like behaviors in mice, J. Neuroinflammation, 14, 190, doi: 10.1186/s12974-017-0964-9.

52. Camargos, Q. M., Silva, B. C., Silva, D. G., Toscano, E. C. B., Oliveira, B. D. S., et al. (2020) Minocycline treatment prevents depression and anxiety-like behaviors and promotes neuroprotection after experimental ischemic stroke, Brain Res. Bull., 155, 1-10, doi: 10.1016/j.brainresbull.2019.11.009.

53. He, L., He, R., Liang, R., Li, Y., Li, X., et al. (2018) Protein expression profiling in the hippocampus after focal cerebral ischemia injury in rats, J. Integr. Neurosci., 17, 149-158, doi: 10.31083/JIN-170047.

54. Radenovic, L., Nenadic, M., Ułamek-Kozioł, M., Januszewski, S., Czuczwar, S. J., et al. (2020) Heterogeneity in brain distribution of activated microglia and astrocytes in a rat ischemic model of Alzheimer's disease after 2 years of survival, Aging (Albany NY), 12, 1225112267, doi: 10.18632/aging.103411.

55. Pluta, R., Ułamek-Kozioł, M., Januszewski, S., and Czuczwar, S. J. (2020) Participation of amyloid and tau protein in neuronal death and neurodegeneration after brain ischemia, Int. J. Mol. Sci., 21, 4599, doi: 10.3390/ ijms21134599.

56. Xu, C. S., Liu, A. C., Chen, J., Pan, Z. Y., Wan, Q., et al. (2015) Overactivation of NR2B-containing NMDA receptors through entorhinal-hippocampal connection initiates accumulation of hyperphosphorylated tau in rat hippocampus after transient middle cerebral artery occlusion, J. Neurochem., 134, 566-577, doi: 10.1111/jnc.13134.

57. Goulay, R., Mena Romo, L., Hol, E. M., and Dijkhuizen, R. M. (2020) From stroke to dementia: a comprehensive review exposing tight interactions between stroke and amyloid- $\beta$ formation, Transl. Stroke Res., 11, 601-614, doi: 10.1007/s12975-019-00755-2.
58. Gemmell, E., Bosomworth, H., Allan, L., Hall, R., Khundakar, A., et al. (2012) Hippocampal neuronal atrophy and cognitive function in delayed poststroke and agingrelated dementias, Stroke, 43, 808-814, doi: 10.1161/ STROKEAHA.111.636498.

59. Hagberg, G., Ihle-Hansen, H., Fure, B., Thommessen, B., Ihle-Hansen, H., et al. (2020) No evidence for amyloid pathology as a key mediator of neurodegeneration poststroke - a seven-year follow-up study, BMC Neurol., 20, 174, doi: 10.1186/s12883-020-01753-w.

60. Hu, Y., Guo, T. C., Zhang, X. Y., Tian, J., and Lu, Y. S. (2019) Paired associative stimulation improves synaptic plasticity and functional outcomes after cerebral ischemia, Neural. Regen. Res., 14, 1968-1976, doi: 10.4103/16735374.259618.

61. Chen, C., Dong, Y., Liu, F., Gao, C., Ji, C., et al. (2020) A Study of antidepressant effect and mechanism on intranasal delivery of BDNF-HA2TAT/AAV to rats with post-stroke depression, Neuropsychiatr. Dis. Treat., 16, 637-649, doi: 10.2147/NDT.S227598.

62. Drapeau, E., Mayo, W., Aurousseau, C., Le Moal, M., Piazza, P. V., and Abrous, D. N. (2003) Spatial memory performances of aged rats in the water maze predict levels of hippocampal neurogenesis, Proc. Natl. Acad. Sci. USA, 100, 14385-14390, doi: 10.1073/pnas.2334169100.

63. Cuartero, M. I., de la Parra, J., Pérez-Ruiz, A., BravoFerrer, I., Durán-Laforet, V., et al. (2019) Abolition of aberrant neurogenesis ameliorates cognitive impairment after stroke in mice, J. Clin. Invest., 129, 1536-1550, doi: 10.1172/JCI120412.

64. Arvidsson, A., Kokaia, Z., and Lindvall, O. (2001) Nmethyl-D-aspartate receptor-mediated increase of neurogenesis in adult rat dentate gyrus following stroke, Eur. J. Neurosci., 14, 10-18, doi: 10.1046/j.0953-816x.2001. 01611.x.

65. Bielefeld, P., Dura, I., Danielewicz, J., Lucassen, P. J., Baekelandt, V., et al. (2019) Insult-induced aberrant hippocampal neurogenesis: Functional consequences and possible therapeutic strategies, Behav. Brain. Res., 372, 112032, doi: 10.1016/j.bbr.2019.112032.

66. Faiz, M., Sachewsky, N., Gascon, S., Bang, K. W., Morshead, C. M., and Nagy, A. (2015) Adult neural stem cells from the subventricular zone give rise to reactive astrocytes in the cortex after stroke, Cell Stem Cell, 17, 624-634, doi: 10.1016/j.stem.2015.08.002.

67. O’Connor, J. C., Lawson, M. A., André, C., Moreau, M., Lestage, J., et al. (2009) Lipopolysaccharide-induced depressive-like behavior is mediated by indoleamine 2,3dioxygenase activation in mice, Mol. Psychiatry, 14, 511522, doi: 10.1038/sj.mp.4002148.

68. Shishkina, G. T., Kalinina, T. S., and Dygalo, N. N. (2007) Up-regulation of tryptophan hydroxylase- 2 mRNA in the rat brain by chronic fluoxetine treatment correlates with its antidepressant effect, Neuroscience, 150, 404-412, doi: 10.1016/j.neuroscience.2007.09.017.

69. Cowen, P. J., and Browning, M. (2015) What has serotonin to do with depression? World Psychiatry, 14, 158-160, doi: 10.1002/wps. 20229 .

70. Shilov, Yu. E., and Bezrukov, M. V. (2013) Kinurenins in the pathogenesis of endogenous mental diseases, Vestn. Ross. Akad. Med. Nauk, 68, 35-41, doi: 10.15690/vramn. v68i1.535. 
71. Tavares, R. G., Tasca, C. I., Santos, C. E., Alves, L. B., Porciúncula, L. O., et al. (2002) Quinolinic acid stimulates synaptosomal glutamate release and inhibits glutamate uptake into astrocytes, Neurochem. Int., 40, 621-627, doi: 10.1016/s0197-0186(01)00133-4.

72. Francija, E., Petrovic, Z., Brkic, Z., Mitic, M., Radulovic, J., Adzic, M. (2019) Disruption of the NMDA receptor GluN2A subunit abolishes inflammation-induced depression, Behav. Brain Res., 359, 550-559, doi: 10.1016/ j.bbr.2018.10.011.

73. Koo, Y. S., Kim, H., Park, J. H., Kim, M. J., Shin, Y. I., et al. (2018) Indoleamine 2,3-dioxygenase-dependent neurotoxic kynurenine metabolism contributes to poststroke depression induced in mice by ischemic stroke along with spatial restraint stress, Oxid. Med. Cell. Longev., 2018, 2413841, doi: 10.1155/2018/2413841.

74. Li, Z. G., Shui, S. F., Han, X. W., and Yan, L. (2020) NLRP10 ablation protects against ischemia/reperfusionassociated brain injury by suppression of neuroinflammation, Exp. Cell Res., 389, 111912, doi: 10.1016/j.yexcr. 2020.111912.
75. Camara, M. L., Corrigan, F., Jaehne, E. J., Jawahar, M. C., Anscomb, H., and Baune, B. T. (2015) Effects of centrally administered etanercept on behavior, microglia, and astrocytes in mice following a peripheral immune challenge, Neuropsychopharmacology, 40, 502-512, doi: 10.1038/npp. 2014.199.

76. Avdic, U., Ahl, M., Oberg, M., and Ekdahl, C. T. (2019) Immune profile in blood following non-convulsive epileptic seizures in rats, Front. Neurol., 10, 701, doi: 10.3389/ fneur.2019.00701.

77. Vieira, M., and Saraiva, M. J. (2014) Transthyretin: a multifaceted protein, Biomol. Concepts, 5, 45-54, doi: 10.1515/ bmc-2013-0038.

78. Sharma, M., Khan, S., Rahman, S., and Singh, L. R. (2019) The extracellular protein, transthyretin is an oxidative stress biomarker, Front. Physiol., 10, 5, doi: 10.3389/ fphys.2019.00005.

79. Ming, Z., Sawicki, G., and Bekar, L. K. (2015) Acute systemic LPS-mediated inflammation induces lasting changes in mouse cortical neuromodulation and behavior, Neurosci. Lett., 590, 96-100, doi: 10.1016/j.neulet.2015.01.081. 